\title{
Strong field processes in beam-beam interactions at the Compact Linear Collider
}

\author{
J. Esberg, ${ }^{1,2}$ U. I. Uggerhøj, ${ }^{1}$ B. Dalena, ${ }^{2}$ and D. Schulte ${ }^{2}$ \\ ${ }^{1}$ Department of Physics and Astronomy, Aarhus University, Ny Munkegade 120, 8000 Aarhus C, Denmark \\ ${ }^{2}$ CERN, CH-1211 Geneva 23, Switzerland
}

(Received 27 January 2013; published 27 May 2014)

\begin{abstract}
The demand for high luminosity in the next generation of linear $e^{+} e^{-}$colliders necessitates extremely dense beams, giving rise to strong fields at the collision point, and therefore the impact of the field on the physical processes occurring at the interaction point must be considered. These processes are well described by the interaction of the individual lepton with the field of the oncoming bunch, and they depend strongly on the beamstrahlung parameter $\Upsilon$ which expresses the field experienced by the lepton in units of the critical field. In this paper, we describe calculations and simulations of strong field processes-also of higher order - at the interaction point.
\end{abstract}

\section{INTRODUCTION}

High precision measurements of possible new physics to be discovered at the CERN LHC are only achievable by a lepton machine due to the noncompositeness nature of the initial particles. In addition to being able to perform precision measurements of the Higgs particle, that measurements have shown exists [1,2], a high center-ofmomentum (CM) electron-positron collider would possess potential for precision measurements of additional exotic particles in the high mass range, and in this respect, high energy is the key. Energy loss to synchrotron radiation dictates that a light lepton $\left(e^{+} e^{-}\right)$collider must be linear if the wall plug efficiency is to be acceptable.

In order to reach significant luminosity, the particle bunches must be very dense. This leads to the presence of strong fields in the rest frame of the particles in the opposing bunch. These fields may approach, or even exceed, the critical field value $\mathcal{E}_{0}=m^{2} c^{3} / e \hbar=1.32 \times 10^{16} \mathrm{~V} / \mathrm{cm}$. Thus, strong field processes may become important, as they, for example, lead to a significant reduction of the beam energy at the time of collision.

The deciding parameter, governing the yield of the strong field processes and the shape of the spectrum of the produced leptons, is the Lorentz invariant quantity $\Upsilon$ defined from

$$
\Upsilon^{2}=\frac{\left(F^{\mu \nu} p_{\nu}\right)^{2}}{m^{2} c^{2} \mathcal{E}_{0}^{2}},
$$

where $F^{\mu \nu}$ is the electromagnetic field tensor and $p^{\mu}\left(\hbar k^{\mu}\right)$ is the four-momentum of the impinging lepton (photon).

Published by the American Physical Society under the terms of the Creative Commons Attribution 3.0 License. Further distribution of this work must maintain attribution to the author(s) and the published article's title, journal citation, and DOI.
In a frame where the field is solely electric and transverse to the particle motion, this reduces to $\Upsilon=\gamma \mathcal{E} / \mathcal{E}_{0}$ where $\gamma=E / m c^{2}$ is the Lorentz factor of the impinging particle ( $\hbar \omega / m c^{2}$ in case of a photon) and $\mathcal{E}$ the local electric field, both measured in the same reference frame, for example, the laboratory. At the Compact Linear Collider (CLIC), an average value of the strong field parameter is $\bar{\Upsilon} \simeq 4$.

The program GUINEA-PIG [3] is one among several codes that can simulate the beam-beam dynamics and background production of a linear collider. The method of simulation is via a particle-in-box approach. Recently, the program was further developed [4] to object oriented $\mathrm{C}++$ code that includes some spin effects and a new method of generating beamstrahlung. The present studies are based on development and usage of this code, with a strong link to experimental results. A more detailed description of a wide range of the topics can be found in [5].

\section{THE CLIC PROJECT}

Two very central keywords in the design of a $e^{+} e^{-}$ collider are energy and luminosity. Nearly all components of such a machine are meant to increase one or both of these. While achieving the energy is often a matter of scaling of components, increasing luminosity for a fixed energy requires a wide range of precision instruments and submachines. In the effort to achieve the required energy and luminosity, the CLIC project strives towards the limiting boundaries of technology and physics in a multitude of ways, with, for example, high frequencies generated by interleaving bunches and a two-beam approach to acceleration. An extensive description can be found in [6] and key parameters can be found in Table 1.

An approximate expression for the instantaneous luminosity one can achieve with Gaussian bunches is 


$$
\mathcal{L}_{0}=\frac{N^{2}}{4 \pi \sigma_{x} \sigma_{y}} f_{\text {rep }} N_{b}
$$

where $N$ is the number of particles per bunch, $\sigma_{x, y}$ are the transverse bunch widths at the interaction point (IP), $f_{\text {rep }}$ is the linac repetition frequency, and $N_{b}$ is the number of bunches per train.

The deciding parameter for strong field effects in a collider is approximately given by [7]

$$
\langle\Upsilon\rangle=\frac{5}{6} \frac{\gamma N r_{e}^{2}}{\alpha \sigma_{z}\left(\sigma_{x}+\sigma_{y}\right)},
$$

in this context known as the beamstrahlung parameter, with $r_{e}$ being the classical electron radius, and $\sigma_{z}$ the length of the beams at the IP. The yield and radiation characteristics of strong field processes depend strongly on the parameter $\Upsilon$. In particular, the spectrum and yield of photons from the IP (beamstrahlung) naturally depends on this parameter.

When Eq. (3) is compared to the expression for the luminosity Eq. (2), one sees that high luminosity and high beamstrahlung parameter are intimately connected.

Since the beam size at the interaction point is $\sigma_{x, y}=\sqrt{\epsilon_{x, y} \beta_{x, y}^{*}}$, where $\beta^{*}$ is the beta function at the IP, the small vertical emittance $\epsilon_{y}$ makes luminosity very sensitive to vertical beam displacements. Furthermore, $\sigma_{y} / \sigma_{x} \ll 1$, in order to keep luminosity (2) high while beam-beam effects relatively low [see Eq. (3)], so the beams are shaped like horizontal sheets of particles. Since $\sigma_{y}=1 \mathrm{~nm}$ at the IP, the final focusing superconducting quadrupole, QD0, must be stabilized with subnanometer precision-an extraordinary technological challenge.
A crossing angle between the beams is necessary with the current time structure of the train of bunches in order to avoid precollisional scattering. Since this means that incoming and outgoing beams are physically separated, the design of postcollision lines is simplified and the feedback and corrector systems are transversely separate. Crab cavities must be used, since the planned crossing angle for CLIC is much larger than the transverse to longitudinal beam ratio, $\sigma_{x} / \sigma_{z} \ll 20 \mathrm{mrad}$. These cavities give the front and rear end of a bunch opposite transverse kicks which rotate the bunch in its average rest frame, recovering luminosity.

If the beta function varies significantly within the bunch length at the IP, the bunches are focused such that they have nominally hourglass-like shapes during interaction. This would also reduce luminosity significantly. However, the effect can, at least in theory, be mitigated by varying the focus of different transverse slices of the bunches, so-called traveling focus. Another option would be to decrease the bunch length in a tradeoff for a larger beamstrahlung parameter, $\Upsilon$.

\section{BEAM-BEAM EFFECTS}

The electric field of one bunch is boosted by the Lorentz factor $\gamma$ when observing it in the laboratory frame. When moving to the rest frame of one bunch, the Lorentz boosted field of the oncoming bunch is enhanced by a factor $2 \gamma^{2}-1$, meaning that immensely strong electromagnetic fields exist in this frame. If the bunches are very dense and energetic, the field of one bunch is able to deflect the oncoming bunch significantly. This force focuses one bunch on the other during crossing, an effect that naturally

TABLE I. $\quad E_{\mathrm{cm}}: \mathrm{CM}$ energy; $\mathcal{L}:$ total luminosity; $\mathcal{L}_{99 \%}:$ luminosity within $1 \%$ of the nominal CM energy; $f_{\text {rep }}$ : repetition frequency; $N_{b}$ : number of bunches per train; $t_{b}$ : time between bunches; $N$ : number of particles per bunch; $\gamma \epsilon_{x, y}$ : horizontal, vertical normalized emittance; $\beta_{x, y}$ : horizontal, vertical beta function at the IP; $\sigma_{x, y, z}$ : horizontal, vertical, longitudinal beam size at the IP; $\delta_{e, p}$ : relative electron/positron energy spread; $\alpha_{c}$ : crossing angle; $f_{\mathrm{rf}}$ : accelerating radio frequency.

\begin{tabular}{lcccc}
\hline \hline & CLIC $3 \mathrm{TeV}$ & CLIC $500 \mathrm{GeV}$ & ILC $500 \mathrm{GeV}$ & ILC $1 \mathrm{TeV}$ \\
\hline$E_{\mathrm{cm}}[\mathrm{GeV}]$ & 3000 & 500 & 500 & 1000 \\
$\mathcal{L}\left[10^{34} \mathrm{~cm}^{-2} \mathrm{~s}^{-1}\right]$ & 5.9 & 2.3 & $1.8^{\mathrm{a}}$ & 4.9 \\
$\mathcal{L}_{99 \%}\left[10^{34} \mathrm{~cm}^{-2} \mathrm{~s}^{-1}\right]$ & 2 & 1.4 & 1.13 & 2.23 \\
$f_{\text {rep }}[\mathrm{Hz}]$ & 50 & 50 & 5 & 4 \\
$N_{b}$ & 312 & 354 & 1312 & 2450 \\
$t_{b}[\mathrm{~ns}]$ & 0.5 & 0.5 & 554 & 366 \\
$N\left[10^{9}\right]$ & 3.72 & 6.8 & 20 & 17.4 \\
$\gamma \epsilon_{x} / \gamma \epsilon_{y}[\mathrm{~nm} \mathrm{rad}]$ & $660 / 20$ & $2400 / 25$ & $10000 / 35$ & $10000 / 30$ \\
$\beta_{x} / \beta_{y}[\mathrm{~mm}]$ & $6.9 / 0.068$ & $8 / 0.1$ & $11 / 0.48$ & $11.0 / 0.23$ \\
$\sigma_{x} / \sigma_{y}[\mathrm{~nm}]$ & $45 / 1$ & $202 / 2.3$ & $474 / 5.9$ & $335 / 2.7$ \\
$\sigma_{z}[\mu \mathrm{m}]$ & 44 & 72 & 300 & 225 \\
$\delta_{e} / \delta_{p}[\%]$ & $0.29 / 0.29$ & $0.35 / 0.35$ & $0.125 / 0.70$ & $0.085 / 0.047$ \\
$\alpha_{c}[\mathrm{mrad}]$ & 20 & 20 & 14 & $20 / 2$ \\
$f_{\mathrm{rf}}[\mathrm{GHz}]$ & 11.994 & 11.994 & 1.3 & 1.3 \\
$\langle\Upsilon\rangle$ & 4.9 & 0.21 & 0.062 & 0.20 \\
\hline \hline
\end{tabular}

${ }^{\mathrm{a}}$ With waist shift, no traveling focus. 
causes growth of the total luminosity. On the other hand the strong radiation resulting from the deflection causes energy loss that means that the mean collision energy is not the double beam energy, but the luminosity becomes a wide spectrum extending to low energies.

The decrease in effective beam size and following enhancement of luminosity can be quantified through the introduction of so-called disruption parameters $[8,9]$

$$
D_{x, y}=\frac{2 N r_{e} \sigma_{z}}{\gamma \sigma_{x, y}\left(\sigma_{x}+\sigma_{y}\right)}
$$

The disruption parameters determine the characteristics of the beam-beam dynamics. The weak disruption regime, when $D \lesssim 0.5$, the intermediate regime when $0.5 \lesssim D \lesssim 5$, and strong disruption is when $D \gtrsim 5$ [9]. In the weak disruption regime, the focal point lies beyond the oncoming bunch, while it moves inside the oncoming bunch in the intermediate regime. In the strong disruption, or "pinching" regime, the central part of one bunch is essentially confined to the oncoming one. In the strong pinching regime, the interaction point may become unstable. One can empirically calculate the beam size as a function of the disruption parameters in the stable disruption regimes.

\section{A. Central background processes}

The total power of the main beam is $14 \mathrm{MW}$ for CLIC at $3 \mathrm{TeV}$ CM. This power should be disposed of properly and valuable information should be extracted from the spent beam as well. Approximately $29 \%$ of the beam power is converted into beamstrahlung photons i.e. about $4 \mathrm{MW}$. They have relatively small polar angles $(<5 \mathrm{mrad})$ and will therefore all exit through the postcollisional beam pipe. Equally, the dominant process of $e^{+} e^{-}$pair production, the coherent pair production, makes the disrupted beam include low energy pairs with angles of emergence from the IP $<10$ mrad; see Fig. 1.

The production of secondary particles has two sides; as well as being a source of background, they provide useful signals for diagnostics, feedback, and luminosity optimization. The primary sources for a fast and direct luminosity signal are the incoherent pairs produced in binary collisions between particles such as $e^{+} e^{-}, e^{+} \gamma, e^{-} \gamma$, or $\gamma \gamma$. They are abundant and their numbers are proportional to luminosity. They have large production angles and thus populate an area of phase space which is otherwise unoccupied. This makes the incoherent pair signal a clean one.

Beamstrahlung from the interaction region has proven a useful diagnostics tool. More specifically it is useful for minimizing the beam sizes at the IP [3]. In the main dump, the physical separation from the charged beam allows for selective conversion of the beamstrahlung photons into muons, that can be counted in a Cherenkov detector behind the main dump. This is a useful signal for monitoring

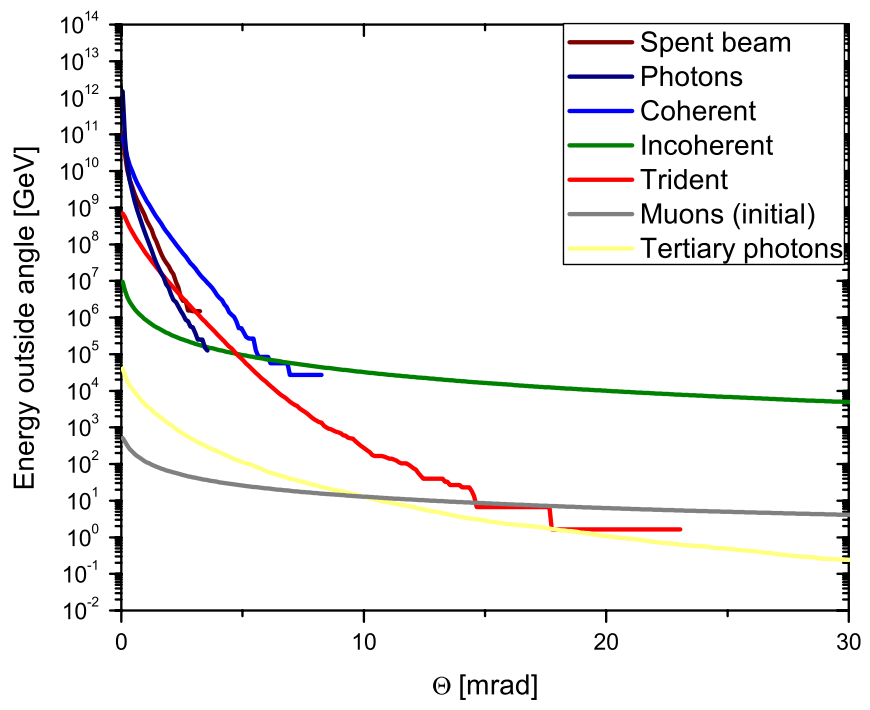

FIG. 1. Energy distributions of the disrupted particles produced at the IP as functions of angular cut. The muons shown here do not include the effect of disruption.

luminosity, and will provide feedback signals for the beam delivery system to maintain high luminosity.

\section{B. Beamstrahlung}

Beamstrahlung, emitted from the IP, is confined to relatively small angles as seen in Fig. 1. In the current versions of GUINEA-PIG and GUINEA-PIG++, the radiation is emitted exactly in the direction of the radiating particle. This is approximately true for very energetic photons, but at very low frequencies $\omega \ll \omega_{c}$, the emission angle is $\theta \approx\left(2 \omega_{c} / \omega\right)^{1 / 3} / \gamma$. The number of photons emitted is approximately $[8,10,11]$

$$
n_{\gamma}=\frac{5}{2} \frac{\alpha^{2}}{r_{e}^{2} \gamma} \frac{\sigma_{z} \Upsilon}{\sqrt{1+\Upsilon^{2 / 3}}}
$$

which is roughly proportional to $N / \sigma_{x}$ for $\Upsilon \ll 1$, where $N$ is the number of particles per bunch, and $\alpha$ is the fine structure constant. Recent experimental investigations [12] have shown that the emission of synchrotronlike radiation in the strong field regime $0.05<\Upsilon<7$ is well described by theory. The generation of beamstrahlung in GUINEAPIG++ closely follows that of CAIN [13], and there is an option to include spin-flip transitions via the SokolovTernov mechanism. In the $\mathrm{C}$ version of the program, spin is not part of the properties of electrons.

\section{Coherent pairs}

Coherent pairs are the pairs generated from the conversion of beamstrahlung photons in the beam-beam field. The number of created coherent pairs in a constant field, applicable in the IP of a collider, is approximately 


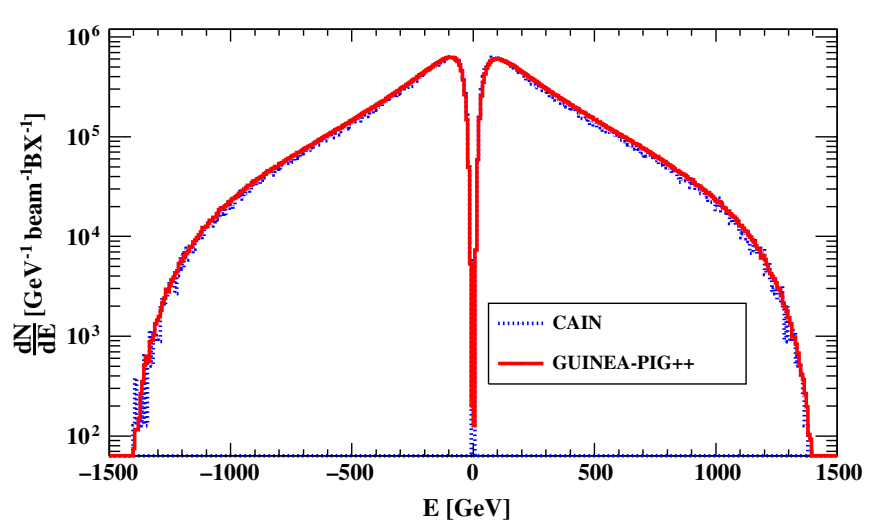

FIG. 2. Comparison between spectra of coherent pairs produced by CAIN and GUINEA-PIG++. The sign of the energy corresponds to the sign of the charge.

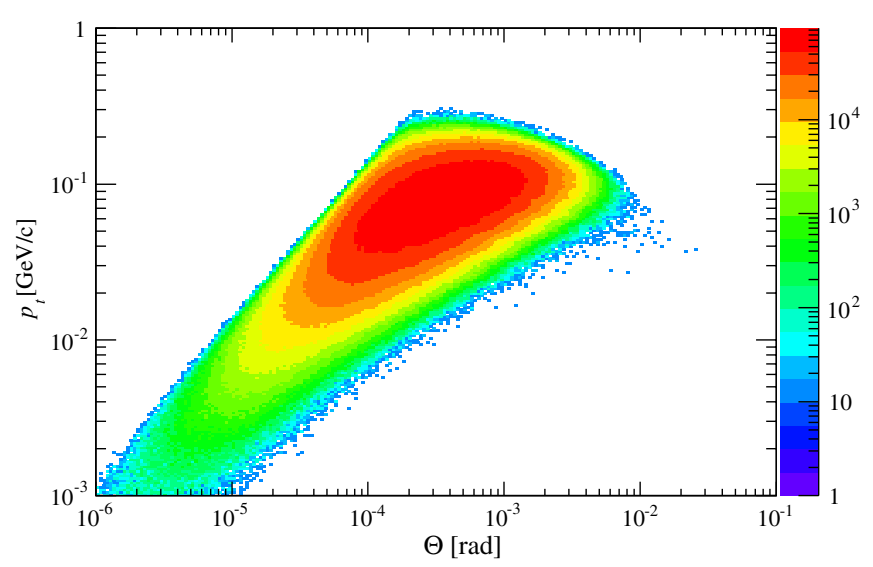

FIG. 3. Distribution of same-charge (defocused) coherent pairs after one bunch crossing using the $3 \mathrm{TeV}$ CLIC baseline design parameters. On the $z$ axis is the number of particles in each bin, per bunch crossing.

$$
n_{p}=\frac{4 \sqrt{3}}{25 \pi}\left(\frac{\alpha \sigma_{z}}{x_{c} \gamma} \Upsilon\right)^{2} \Xi(\Upsilon)
$$

where several expressions for the auxillary function $\Xi$ exist [8,14-16]. Chen and Telnov [15] give the approximate expression

$$
\begin{gathered}
\Xi(\Upsilon)=0.5 \exp [-16 /(3 \Upsilon)], \quad \Upsilon \ll 1, \\
\Xi(\Upsilon)=2.6 \Upsilon^{-2 / 3} \ln (\Upsilon), \quad \Upsilon \gg 1,
\end{gathered}
$$

where $\chi=\hbar / m c$ is the reduced Compton wavelength. The quadratic dependence on the bunch length for a fixed $\Upsilon$ reflects the fact that photons creating the pairs must be created before the photon can convert. Generation of pairs in GUINEA-PIG++ once again closely follows the implementation in CAIN [13]. As can be seen in Fig. 2, the two programs agree on the spectra of produced coherent pairs.

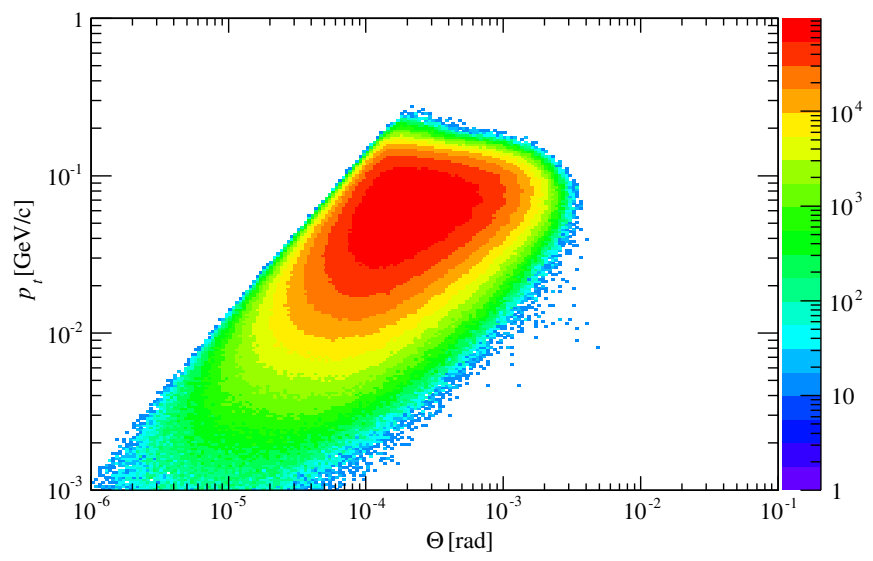

FIG. 4. Distribution of opposite-charge (focused) coherent pairs after one bunch crossing using the $3 \mathrm{TeV}$ CLIC baseline design parameters. On the $z$ axis is the number of particles in each bin, per bunch crossing.

This is a valuable cross-check of the generators of coherent pairs as well as of bremsstrahlung photons.

The kinematic distribution of the coherent pairs can be seen in Figs. 3 and 4. The angles of these particles are relatively well confined meaning that cutting away these particles in the postcollisional line is achievable. The sign of the charge of the produced particles determines weather it is defocused or pinched by the oncoming beam which then in turn determines the postcollitional characteristics of the occupied phase space. The particles with the same charge as the opposing beam reach the largest angles due to the defocusing nature of the force they experience. These particles emerge from the IP with maximum polar angles of approximately $\sqrt{4 \ln D_{x} / \epsilon+1 D_{x} \sigma_{x}^{2} /\left(\sqrt{3} \sigma_{z}^{2} \epsilon\right)}$ [3] with $\epsilon$ being the particle energy relative to the beam energy.

\section{TRIDENTS}

Because the QED critical field is of a magnitude such that an electron will gain an energy of $m c^{2}$ by moving a reduced Compton wavelength, it is not surprising that spontaneous emission of pairs by an electron in such a field is expected. One could say that virtual pairs associated with the electron field will be able to reach the mass shell in fields of such magnitudes. The direct trident process $e^{-}+\mathcal{E} \rightarrow e^{-}+\mathcal{E}+e^{-}+e^{+}$represented by the Feynman diagram in Fig. 5 is the emission of an electron/positron pair in a strong field [17] without intermediate steps.

Events where an electron produces a pair in the interaction with an electromagnetic field may proceed either through the sequential process where the electron emits a real photon that converts into a pair, or directly where the intermediate stage contains a virtual photon. A recent measurement of trident events from both amorphous and crystalline matter may be found in [18]. In fact, energetic 


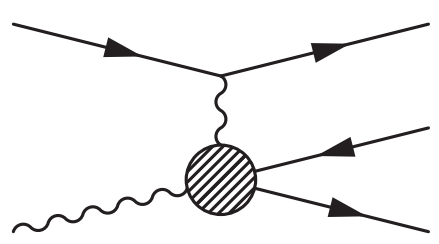

FIG. 5. Feynman diagram for the direct trident process used for the Weizsäcker-Williams calculation. The incoming wavy line represents the coherent interaction of many particles-described as a homogeneous field - with the electron.

particles interacting with an oncoming crystal (as seen from the frame of the particle) closely resembles the situation of particles interacting with an oncoming bunch. As stated by Blankenbecler and Drell in their seminal paper [19] "...we find it convenient to work in the rest frame of the pulse which transforms into a very long narrow 'string' of $N$ charges," directly reminiscent of the string of charges constituting a crystal. An introduction to particle interactions in crystalline matter at high energies can be found in [20].

Many conventions for the naming of produced pairs exist - especially when not only interactions with the field are considered. The term "trident" may refer only to the direct trident process, also known as magnetic electroproduction, but in some contexts it refers to the union of the sets "sequential tridents" and "direct tridents" due to the three-prong track of the reaction products when they are subject to a magnetic field.

In GUINEA-PIG "incoherent pairs" refers to pairs produced in binary collisions. One of the possibilities for a pair producing collision is one between two leptons i.e. the Landau-Lifshitz process. This looks strikingly similar to the amorphous trident production mechanism via a virtual photon. However, incoherent pairs also include photon-photon or photon-lepton collisions. All these processes are shown in Fig. 6.

Here we will distinguish between direct tridents (magnetic electroproduction) and sequential tridents (coherent pairs). Finally, "incoherent tridents" means Landau-Lifshitz-like pairs, i.e. resulting from binary collisions between two leptons - or in material media between a nucleus and a lepton.
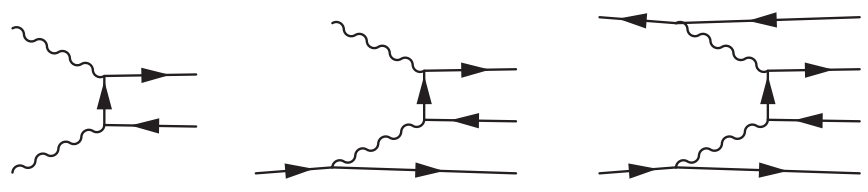

FIG. 6. Feynman diagrams for incoherent pairs and incoherent muons. From left to right: the Breit-Wheeler, Bethe-Heitler, and Landau-Lifshitz processes. For each of these $u$-channel diagrams, there is a corresponding $t$-channel diagram (which means connecting each photon to the opposite virtual fermion vertex).

\section{A. Direct tridents in colliders}

The sequential trident events are important when considering the design of a TeV scale linear $e^{+} e^{-}$collider: they are the result of beamstrahlung photons converting in the strong field of the oncoming bunch and may contribute $\sim 10 \%$ of the total charge after the bunch crossing. Likewise, the result of the conversion of virtual photons in the field of the oncoming bunch, direct trident events, may be significant and can be analyzed using a WeizsäckerWilliams calculation.

The subject of equivalent-quanta trident production in a homogenous field has been studied by several authors [21] or in crystals using the Weizsäcker-Williams method [22]. Decreasing the bunch lengths in order to minimize the hourglass effect could be an attractive way of increasing luminosity when the bunches are tightly focused. However, since the strong field parameter is proportional to $1 / \sigma_{z}$ this would increase the strong field parameter making the quantum nature of the IP more pronounced. The direct trident process depends strongly on the strong field parameter and could potentially become a dominant channel for pair creation. Furthermore, the emitted electrons and positrons can be of rather low energies [23] compared with coherent pairs, which means that they could emerge from the IP with rather large angles.

Not many analytical expressions for the yield exist [15,23-26], and to the knowledge of the authors, a single conversion probability in closed form, valid in the intermediate range $\Upsilon \approx 1$ does not exist. However, estimates do exist and according to $[15,27]$ the total number of created direct tridents is

$$
n_{\mathrm{tr}}=\frac{4 \sqrt{3}}{25 \pi}\left(\frac{\alpha \sigma_{z} \Upsilon}{\gamma \chi_{c}}\right) \Omega(\Upsilon)
$$

where $\sigma_{z}$ is the bunch length and

$$
\Omega(\Upsilon)=2.6 \alpha \ln (\Upsilon), \quad \Upsilon \gg 1
$$

This expression, however, fails at small energies. In order to mitigate, we here use a Weizsäcker-Williams calculation [28].

The spectrum of virtual photons can be estimated to first order using $[28,29]$

$$
\frac{\mathrm{d} n_{v}}{\mathrm{~d} x}=\frac{\alpha}{2 \pi} \frac{1+(1-x)^{2}}{x} \ln \left(\frac{q_{\max }^{2}}{q_{\min }^{2}}\right),
$$

$x=\hbar \omega / E_{0}, E_{0}$ being the energy of the emitting particle. An integration of the virtual photon spectrum from $q_{\min }^{2}$ to $q_{\max }^{2}$ has been done creating a cutoff at these virtualities. For the purpose of this article and the GUINEA-PIG++ calculations, this spectrum is used with $q_{\max }^{2}=m^{2}$ and $\left|q_{\min }\right|^{2}=x^{2} m^{2}$ [25]; see Fig. 7 . 


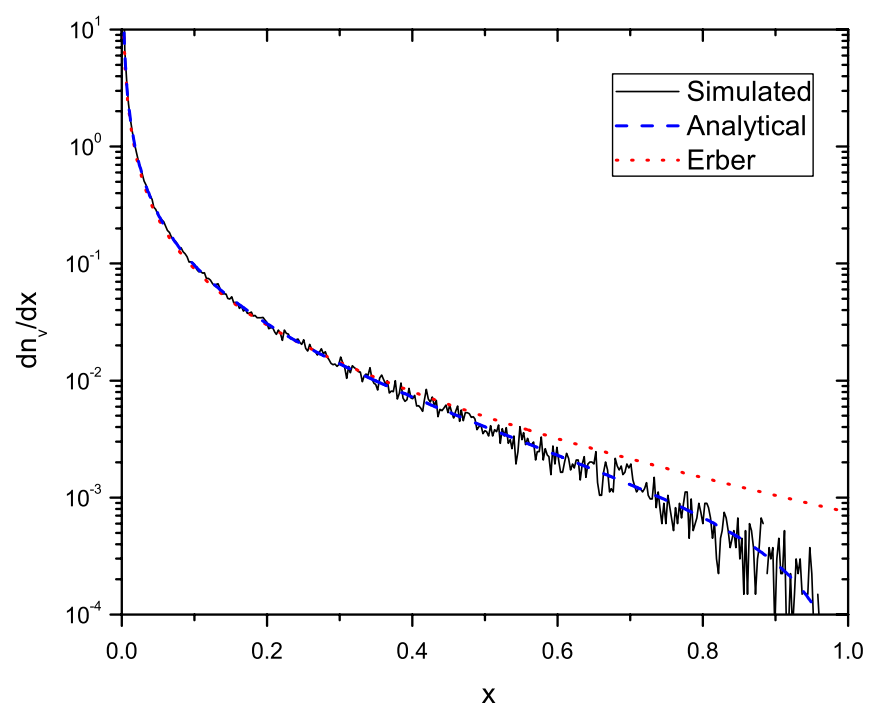

FIG. 7. Spectrum of virtual photons used for generating direct tridents, $x=\hbar \omega / E_{0}$. The expression (11) ("analytical") exhibits a cutoff in the maximum energy, which is clearly more physical than the semiclassical spectrum ("Erber") from [14].

The total number of virtual photons carried by an electron is then approximately $n_{v} \approx \alpha \ln ^{2}\left(x_{\min }\right) / \pi$ for $x_{\min } \ll 1$. For the sake of calculations $x_{\min }=10^{-5}$ was chosen as a default, ensuring that the simulation is valid for all currently considered collider designs since the smallest energy of the produced particles is higher than this fraction of the primary electron energy [23].

Since the relevant virtualities are very small, the virtual photon has near on-shell properties. Therefore it can be assumed to convert in the external field with the probability and spectrum of an unpolarized photon on the mass shell, $q^{2}=0$. The total cross section can be parametrized by [13]

$$
p=0.23 \alpha \frac{m c^{2}}{\hbar \omega} \frac{\Delta z}{\lambda_{c}} \kappa e^{-8 / 3 \kappa}(1+0.22 \kappa)^{-1 / 3},
$$

where $\kappa=x \Upsilon$, the strong field parameter of the photon, while the photon converts with the differential probability [24]

$$
\begin{aligned}
\frac{\mathrm{d} p}{\mathrm{~d} x^{\prime}}= & \frac{\alpha}{\sqrt{3} \pi} \frac{\Delta z}{\lambda_{c}} \frac{m c^{2}}{\hbar \omega} \\
& \times\left(\frac{x^{\prime 2}+\left(1-x^{\prime}\right)^{2}}{x^{\prime}\left(1-x^{\prime}\right)} K_{2 / 3}(\xi)+\int_{\xi}^{\infty} K_{1 / 3}(y) \mathrm{d} y\right),
\end{aligned}
$$

where $x^{\prime}=E_{-} / \hbar \omega$ is the electron fraction of the photon energy and $\xi=\frac{2}{3 \kappa} \frac{1}{x^{\prime}\left(1-x^{\prime}\right)}$. Also Eq. (13) has been tested successfully by experiments utilizing crystalline targets $[30,31]$.

Special numerical techniques are needed to sample from this function. An easily invertible approximate expression for the integral of this function can be used to determine the sampled energy [13], while the real expression (13) is used to correct the cross section. This method of determining the energy follows the method described in [13]. For Monte Carlo simulation purposes, the exact expression for the differential probability is evaluated using interpolations of the modified Bessel functions $K_{2 / 3}$ and the integral of $K_{1 / 3}$.

In extreme fields, one finds corrections to the virtual photon spectrum. Here, we only mention the possible corrections without applying them to real simulations. The maximum magnitude of the virtuality chosen above is the squared center-of-mass energy. An additional correction to the minimal virtuality arises from the recoil of the electron $q_{\min }^{2} \approx m^{2}\left(x^{2} \Upsilon\right)^{2 / 3}$ when $(\Upsilon / x)^{1 / 3} \gg 1$. This effect gives rise to the mechanism of magnetic suppression, for example, relevant for bremsstrahlung [32], but this cut is not used for the direct trident generation.

Because of the continuous nature of the process, the probability of photon conversion in a single step is attenuated when the linear probability gets large

$$
p^{\prime}=1-e^{-p} .
$$

This means that in simulations with large $\Upsilon$, the step size should not be too large to ensure that a linear expansion of (14) is valid.

In order to determine any effects on the beam particles, the energy of the producing electron is modified if the virtual photon converts, and the direct tridents are added to the luminosity calculation as well as the bunch field calculation of GUINEA-PIG++.

\section{B. Results of simulation}

A comparison between a version of the direct trident generator with constant $\Upsilon$ and expressions from [14,15] is seen in Fig. 8. Clearly, the approximate expressions are not adequate for determining the yield at the threshold for quantum effects around $\Upsilon=1$. In Erber's seminal paper [14] a semiclassical Weizsäcker-Williams approximation is used for the virtual photon spectrum. This spectrum weighs more on high energies than Eq. (11) which explains the difference at $\Upsilon<1$ and the similarity at $\Upsilon \gg 1$. The spectra coincide completely at low energies as can be verified analytically. The Weizsäcker-Williams approximation used here is much better in the intermediate range besides being valid in the quantum regime.

Figures 11, 12, and 9 show the results of a full CLIC $3 \mathrm{TeV}$ simulation using realistic input distributions. The amount of direct tridents is $3.6 \times 10^{6}$ per beam per bunch crossing or approximately one per mil of the total initial bunch charge. By comparison, the coherent pairs contribute with 2 orders of magnitude more charge. The smallness of the yield of direct tridents means that the parameter region, where this ratio is 1 , is quite far from any realistic CLIC parameter set. 


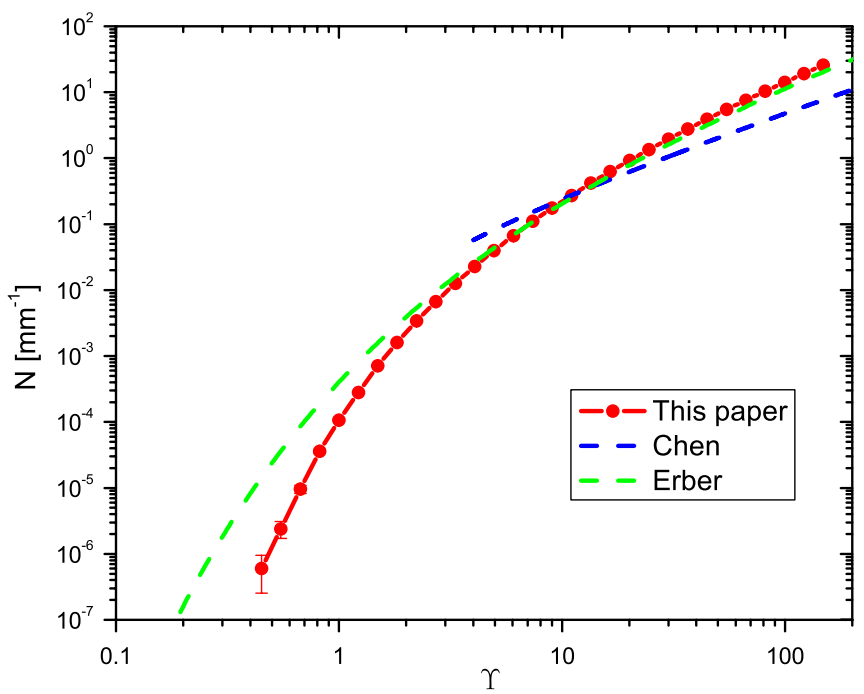

FIG. 8. Total yield of direct tridents at a primary energy of $250 \mathrm{GeV}$. Red: Monte Carlo simulation, this paper. Blue dashed: approximate expression by Chen [15]. Green dotted: Erber [14]. Note that the Chen and Erber curves are shown outside of their respective regions of validity.

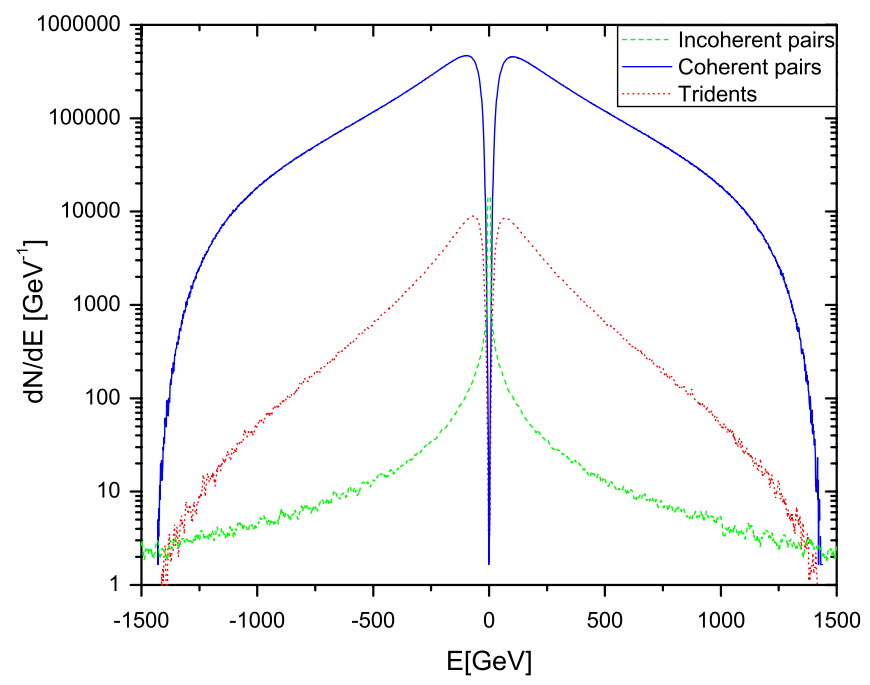

FIG. 9. Spectrum of the various pair production mechanisms after full beam crossing simulation.

The development of the yield of the direct tridents closely follows that of the coherent pairs with respect to a vertical offset of the beams since they are both strong field processes. This means that vertical offsets will not vary the relative composition of produced strong field pairs significantly.

This study, using Gaussian beams in the CLIC nominal parameter range, is seen in Fig. 10. Here, it becomes evident that the bunches must become prohibitively short if direct tridents are to be produced in large numbers.

The incoherent pair production mechanism produces approximately $1.5 \times 10^{5}$ particles per beam per bunch

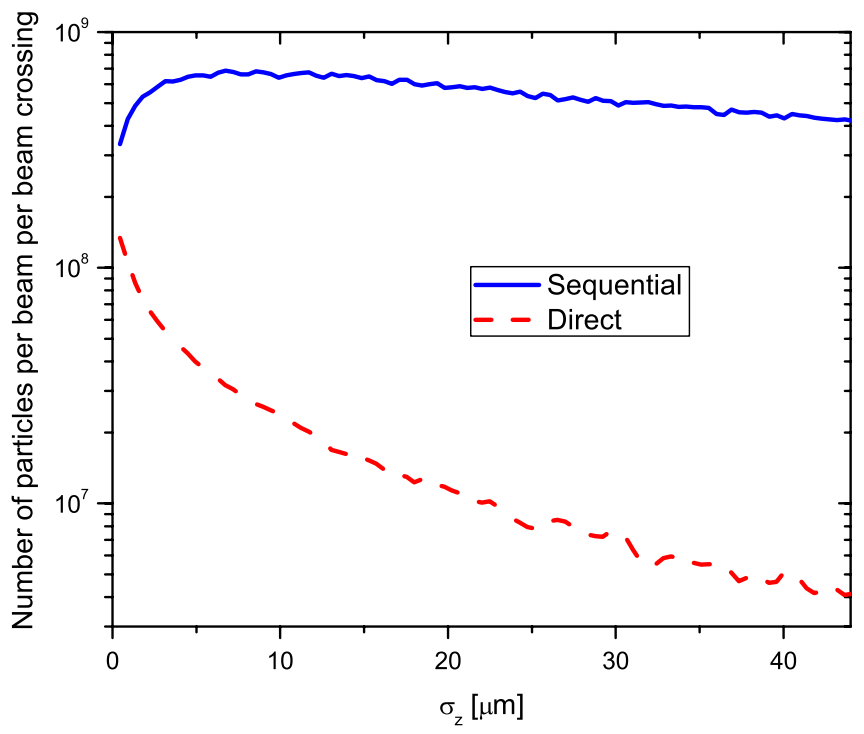

FIG. 10. Bunch length dependence of the yield of the coherent pair production mechanisms. The bunch charge is kept constant.

crossing. Because of the nature of the process and since the spectrum is peaked at energies just above the electron mass, these particles can emerge from the interaction region at very large angles. This makes them suitable for luminosity monitoring. The large difference in the spectrum and angles of these pairs in comparison to the direct trident process lies in the assumption of complete field coherence in the production of a direct trident. If, however, a single electron interacts coherently with several particles, but not a continuous field, neither of these models for pair production will be sufficient. In the laboratory frame, the density of electrons/positrons is of the same order as in solid matter, which means that the scattering centers are close.

The angular distribution of the direct tridents is narrower than that of the incoherent pairs and the energies not as low as those of the incoherent pairs. Even so, there was an area of phase space that the coherent pairs did not occupy, while the same area was populated by incoherent pairs. This, before the tridents were simulated, allowed for an almost complete separation of these pairs by positioning the lumical at a certain polar angle. The direct tridents narrow the window in angle $/ p_{t}$, meaning that they should be kept in mind when designing the forward detector which is intended for luminosity monitoring utilizing incoherent pairs [33].

Thus, a generator of direct trident that is valid in the quantum mechanical as well as in the intermediate $\Upsilon \approx 1$ regime has been developed. This generator has been applied to beam-beam simulations relevant for CLIC. The impact on any proposed $3 \mathrm{TeV}$ CLIC design is small when compared to other processes of $e^{+} e^{-}$pair creation but may become significant in the limit of short bunches. The kinematic characteristics of the produced particles are not unlike those of sequential tridents. Since the relative 


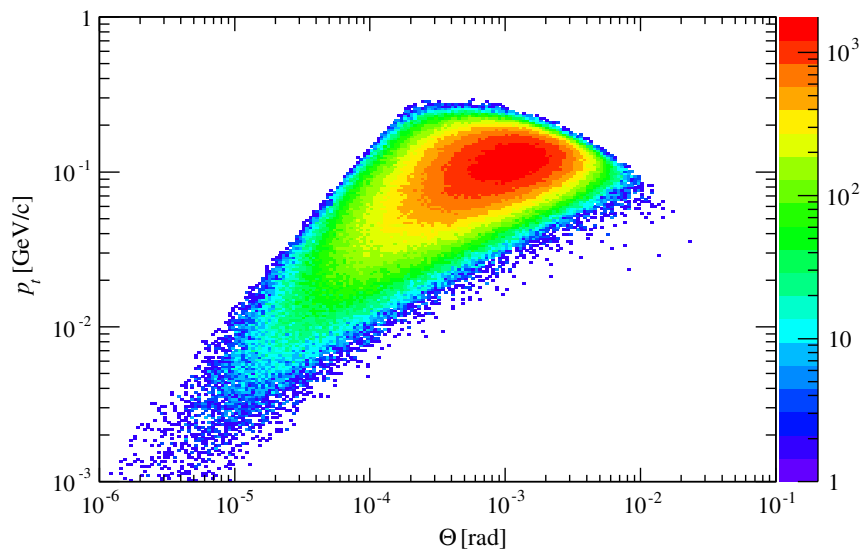

FIG. 11. Distribution of same-charge (defocused) direct tridents after one bunch crossing using the $3 \mathrm{TeV}$ CLIC baseline design parameters. On the $z$ axis is the number of particles in each bin, per bunch crossing. May be compared with the distribution of coherent pairs shown in Figs. 3 and 4.

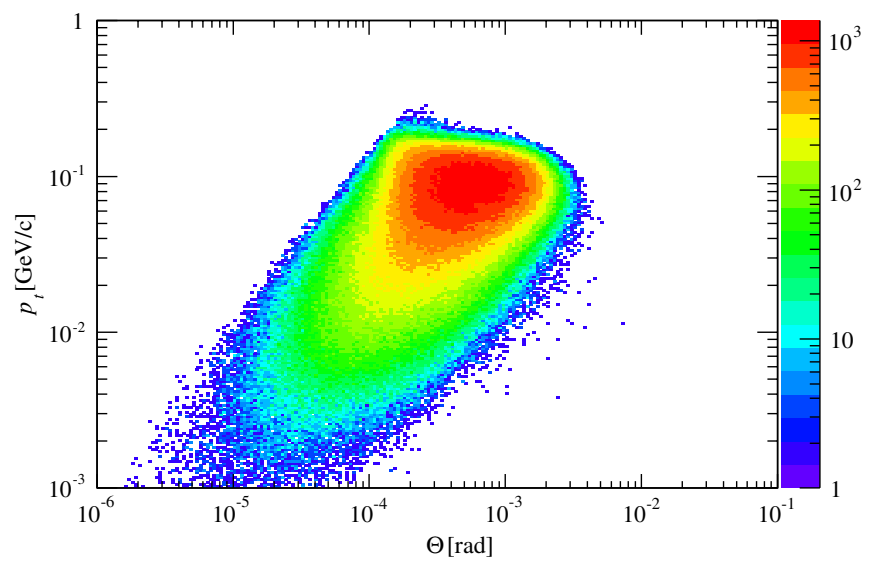

FIG. 12. Same as Fig. 11 but for opposite-charge (focused) particles.

contribution of the direct tridents to the postcollisional bunch charge is small, this will mean that they are most likely difficult to distinguish from the coherent pairs in a detector. Varying the bunch length will, however, alter the ratio of the yields of these processes.

The physics performance potential of the detector is limited by background processes. Beam-beam induced backgrounds include minijets from $\gamma-\gamma$ collisions, coherent pairs, and incoherent pairs as well as incoherent muons and direct trident pairs. The presence of these processes influences the design of the detector and the event selection algorithms in various ways. A great number of these processes are implemented in GUINEA-PIG and GUINEA-PIG++. They include Bhahba scattered particles and radiative Bhabha events, bremsstrahlung photons, minijets, and initial state radiation [34].

The strong field effects at the IP may further modify the vertices of key physics processes, for example, $W$ pair production. This process may be useful in polarimetry measurements [35] if one measures the left-right asymmetry of the produced $W$ 's. The strong field effects on these matters have not been extensively studied.

\section{LUMINOSITY SPECTRUM}

The energy loss due to beamstrahlung-and other mechanisms - means that a substantial fraction of collisions will occur at lower energies than the nominal $\mathrm{CM}$ energy. Furthermore, collisions involving coherent pairs will contribute to luminosity at lower energies. The luminosity spectrum is seen in Fig. 13. The quantum nature of beamstrahlung is naturally very important to take into account. The ratio of the luminosity within $1 \%$ of the nominal center-of-mass energy to the total luminosity is $34 \%$, while the ratio would be in the vicinity of $17 \%$ if the particles radiated with the classical synchrotron probability. This very significant difference has recently been corroborated by experiment [12].

\section{A. Properties of produced particles}

The simulated production angles of various beam particles can be seen in Fig. 1; see, for example, also [34]. This provides some information of the dynamics of each single process. Generally, incoherent processes extend to large angles since the center-of-mass frame is approximately the laboratory.

During active stabilization of the beams, rather large offsets of the beams with respect to one another can be expected. Luminosity and effective fields experienced by a beam particle are in particular very sensitive to vertical offsets since the beams are "flat." The change of amount of produced particles with this offset then gives insight to the instantaneous luminosity. The behavior of some yields can be seen in Fig. 14. For coherently produced particles,

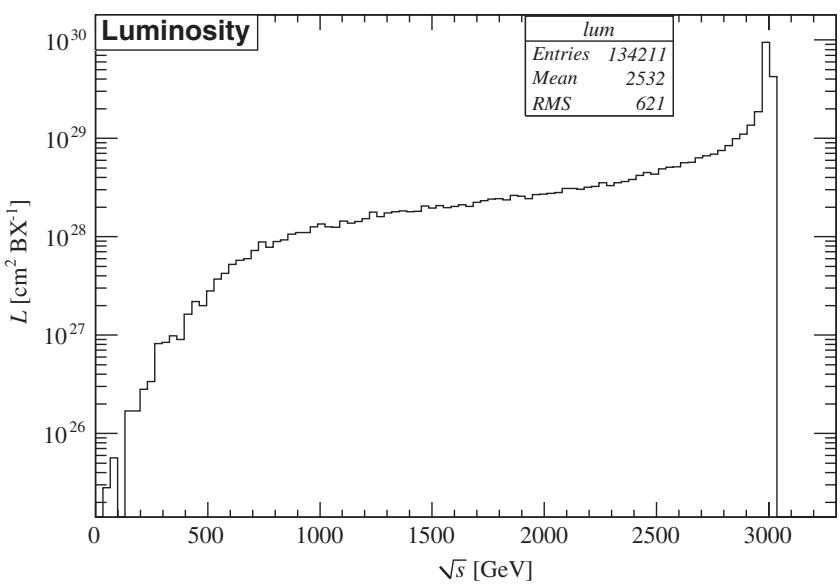

FIG. 13. CLIC nominal luminosity spectrum at ideal conditions; the spectrum includes contributions from coherent pairs and initial state radiation. 


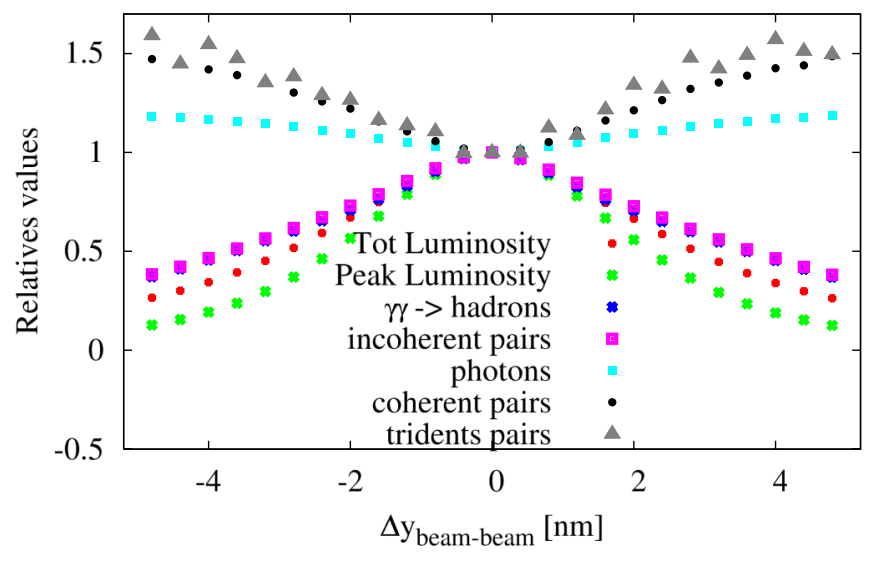

FIG. 14. Dependence of various relative yields on vertical offset between colliding bunches normalized to production at perfect conditions. The average strong field increases with this offset, increasing the yield of strong field processes. Peak luminosity refers to the amount of luminosity within $1 \%$ of the nominal collision energy. $\gamma \gamma \rightarrow$ hadrons refers to the amount of hadronic events from collisions between real or virtual photons.

the yield increases with offset due to increase in the field while the incoherently produced particle yields decrease. If coherently produced charged particles were to be used as luminosity signals, spectral information is furthermore needed. This is so, since the spectra change significantly with the field which in turn will affect the detection efficiency.

\section{DEPOLARIZATION}

Ideally, the colliding beams of a future collider should be longitudinally polarized, meaning that the spin vectors of beam particles should have an average longitudinal direction in space. The strong field beam-beam effects could potentially partially destroy this polarization, partly due to the deflection of the beam particles that will cause strong spin precession, and partly due to the radiative spin-flip process.

Electron sources that utilize the band structure of gallium arsenide (GaAs) will be able to produce highly polarized beams when struck by circularly polarized photons. Further refinements of such sources that introduce a periodic deformation of the surface-a so-called super latticepromise to be able to produce highly polarized ( $\sim 90 \%)$ electrons [36].

A possible source of positrons would utilize a storage ring able to Compton-backscatter photons. This gives rise to photons of high energy and high degree of polarization. The scattered photons would then be converted to pairs using a solid target that could possibly be crystalline [37].

The spin should be vertical before the particles are entering damping rings and the ring to main linac line, since the subsequent magnetic deflection would otherwise cause spin precession. This is achieved in spin rotators that utilize the spin precession to orient the average spin. Before the main linac, the spin is intended to be rotated back to the longitudinal direction of the main linac for the collisions.

\section{A. The T-BMT equation}

The T-BMT equation $[38,39]$ describes the dynamics of the classical spin in a homogeneous electromagnetic field.

If this mechanism would be the only one governing the spin dynamics of the interaction point, total depolarization would be $\langle D\rangle=(\gamma a)^{2} / 2\left[\Delta \theta_{x, \mathrm{rms}}^{2}+\Delta \theta_{y, \text { rms }}^{2}\right]$ where $\Delta \theta_{\text {rms }}$ is the rms deflection angle of the beam particles and $a$ is the anomalous magnetic moment of the electron [40]. Since $\Delta \theta_{\mathrm{rms}} \approx 50 \mu \mathrm{rad}$ [41], we would expect an average depolarization of approximately $6 \%$ from spin precession alone. However, the strong field greatly reduces the magnitude of depolarization from this mechanism.

\section{B. High field correction to the anomalous magnetic moment}

Although not very well known, the anomalous magnetic moment of the electron, $a$, is not a universal constant [42]. In strong fields this quantity is modified due to a change in the mass operator. The theoretical value of $a$ is changed to

$a(\Upsilon)=\frac{\alpha}{2 \pi} \int_{0}^{\infty} \int_{0}^{\infty} \frac{v}{1+v^{3}} \sin \left(\frac{v}{\Upsilon}\left[z+\frac{z^{3}}{3}\right]\right) \mathrm{d} v \mathrm{~d} z$,

or approximately

$$
a(\Upsilon)=\left\{\begin{array}{ll}
\frac{\alpha}{2 \pi}\left(1-12 \Upsilon^{2}\left[\ln \left(\frac{1}{\Upsilon}\right)-1.957\right]\right) & \Upsilon \ll 1 \\
\frac{\alpha}{9 \sqrt{3}} \Gamma(1 / 3)(3 \Upsilon)^{-2 / 3}\left(1+6 \frac{\Gamma(2 / 3)}{\Gamma(1 / 3)(3 \Upsilon)^{-2 / 3}}\right) & \Upsilon \gg 1
\end{array} .\right.
$$

GUINEA-PIG++ and CAIN use more elaborate, but identical, approximate expressions to calculate the anomalous magnetic moment on the fly. These expressions coincide when $\Upsilon=0.6125$. So for $\Upsilon$ above this value the lower expression is used while the upper expression is used for calculation of the magnetic moment when $\Upsilon>0.6125$. The function implemented in these programs can be seen in Fig. 15. If the magnetic moment were constant, the majority of the T-BMT spin precession would take place in regions of high fields, but the nonlinear reduction of $a$ counteracts this effect meaning that the spin precession part of the depolarization in the CLIC interaction region is significantly suppressed.

\section{Sokolov-Ternov spin flip}

Another source of depolarization is the radiation emission. When $\Upsilon$ becomes of the order one, a significant portion of the radiation emission originates from the 


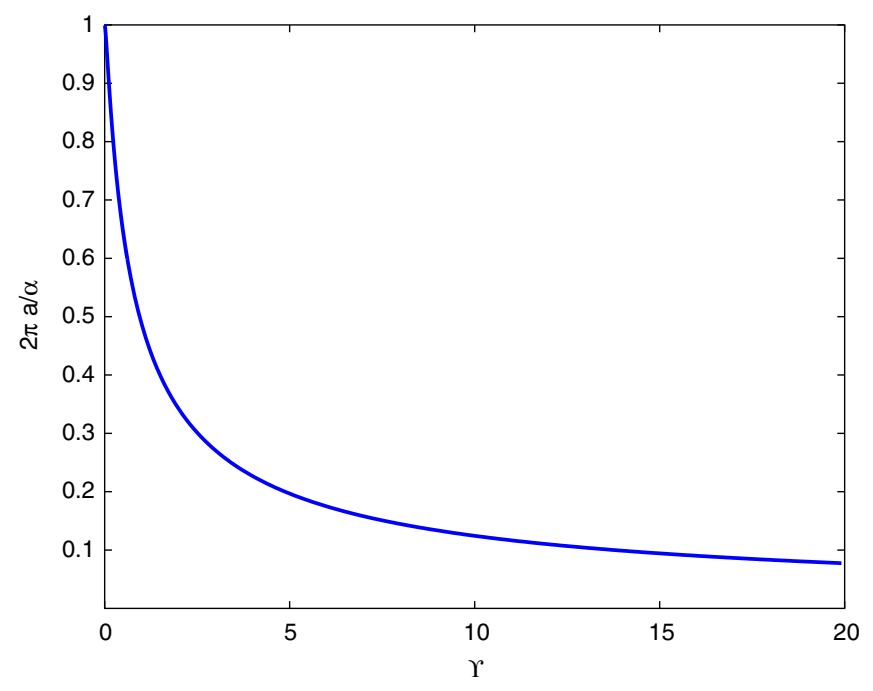

FIG. 15. The strong field dependence (16) of the anomalous magnetic moment of the electron.

electron reversing its spin. The already existing implementation of the spin-flip process on the radiating electron in GUINEA-PIG++ is very closely related to the one described in the CAIN manual [13]. It has been confirmed that the implementation reproduces the result described in [43], even though there was until recently a problem with the normalization of the spin vector. The significance of the spin-flip process in strong fields has also been observed experimentally in crystals [44].

An estimate for the beam-beam depolarization including the Sokolov-Ternov process is given by Yokoya and Chen in [40]. Using analytic expressions from this article, one can estimate the total depolarization due to spin-flip radiation to approximately $8.5 \%$ when one takes the deflection parameters into account [their Eq. (45)]. When $\Upsilon \ll 1$, this article furthermore gives the convenient estimate for the Sololov-Ternov driven depolarization as $(7 / 27) n_{\gamma}\langle\Upsilon\rangle^{2}$, where $n_{\gamma}$ is the number of synchrotron photons per beam particle, but since the low-field condition is not fulfilled for CLIC this estimate has not gotten much use in this case.

\section{Simulations of depolarization for CLIC}

When doing simulations using GUINEA-PIG++ and using realistic input beams, one gets the total depolarizations listed in Table II. In this case depolarization for the spent beams is defined as $1-\left\langle S_{z}\right\rangle$ while the luminosity weighted depolarization is calculated as $1-\sqrt{\left\langle S_{z 1} S_{z 2}\right\rangle}$. Here, $S_{z}$ are the longitudinal components of the spin vector $\left(\left|S_{z}\right|=1\right)$ and the numerical indices correspond to the bunch to which the colliding particle belongs. The numbers presented here have been cross-checked with the results obtained in [43], and found to agree.

Because the Sokolov-Ternov (ST) mechanism dominates the CLIC depolarization, one would expect that the
TABLE II. Simulated total depolarizations for CLIC 3 TeV. The beams are assumed to be completely longitudinally polarized before collision.

\begin{tabular}{lcc}
\hline \hline Total depolarizations & BMT & BMT + ST \\
\hline Spent beam & $0.33 \%$ & $6 \%$ \\
Luminosity weighted & $8.9 \mathrm{e}-2 \%$ & $3 \%$ \\
\hline \hline
\end{tabular}

particles least influenced by synchrotron radiation are the ones that are least depolarized $[33,45]$. This tendency can be seen in Fig. 16, where it is apparent that the energy dependence

of depolarization is strong. If one is able to isolate the collisions occurring within the $1 \%$ of nominal center-ofmass energy, the luminosity weighted depolarization for these collisions is reduced to only $0.5 \%$, an almost negligible number compared to the few percent that one would expect for the total depolarization.

Since the deflection and mean strong field parameter increases with offset between the colliding beams, one would expect the depolarization to increase accordingly. In Fig. 17 the offset dependence of depolarization is shown to be rising significantly with vertical offset, but in the $1 \%$ luminosity peak, the luminosity weighted depolarization remains relatively small although not entirely negligible anymore with offsets in the order of a few nanometers.

In conclusion, the nonlinear strong field effects at the interaction point tend to favor depolarizations that are quite small. And in the interesting energy region, the depolarization is significantly smaller than estimated in analytical formulas [40]. This means that other sources, for example, the beam delivery system, are likely to account for an equal or greater degree of depolarization of the luminosity peak in a linear collider operating in the strong field regime.

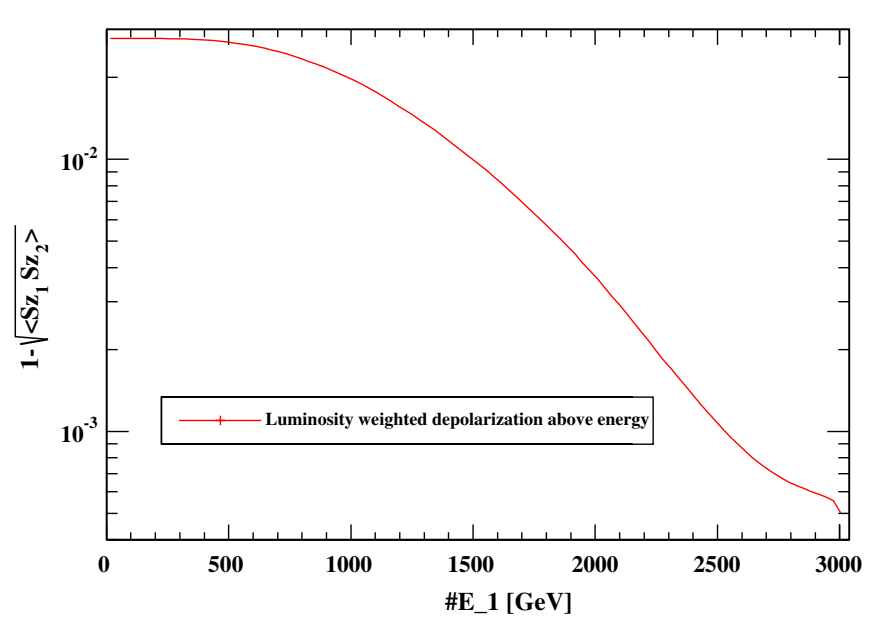

FIG. 16. The CLIC depolarization as function of a cut in the lower CM energy. 


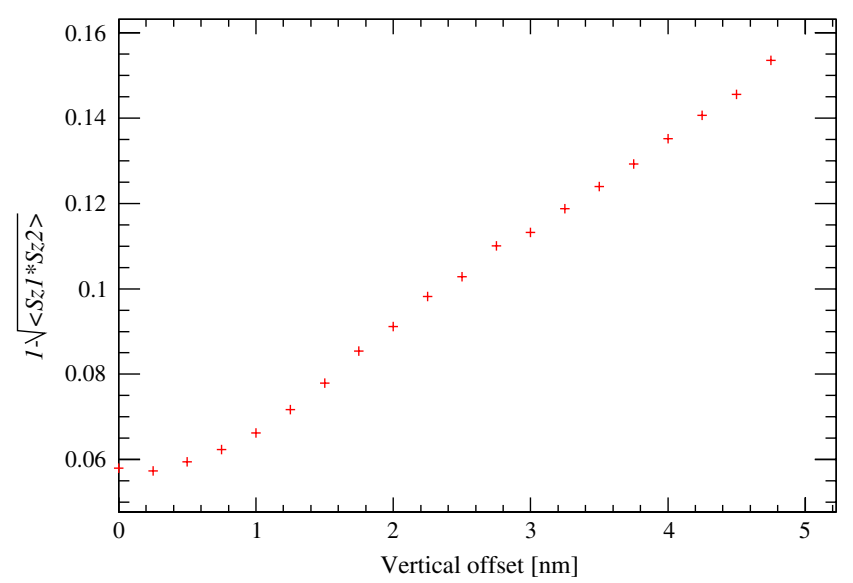

background events in the muon chambers surrounding the detectors. Muon pairs will however also be produced incoherently, where the virtual lepton is a muon. They extend to rather large angles creating background muon signals. This makes knowledge of their abundance and kinematics important.

\section{A. Method of generation}

Muons are generated almost identically to the incoherent pairs in GUINEA-PIG++ as in Fig. 6 where the only difference is their mass. This description of the muon generator here will follow the one in [3]. The Breit-Wheeler process where two photons collide can be simulated while the Bethe-Heitler (colliding one photon and one lepton) and Landau-Lifshitz (colliding two leptons) processes can be done utilizing a Weizsäcker-Williams calculation replacing the lepton by its virtual photon flux.

This procedure gives the well-known total cross section of

$$
\begin{aligned}
\sigma_{\mathrm{BW}}= & \frac{\pi r_{\mu}^{2} m_{\mu}^{2} c^{2}}{s} \\
& \times\left[\left(2+\frac{2}{\gamma^{2}}-\frac{1}{\gamma^{4}}\right) \ln \left(\frac{1+\beta}{1-\beta}\right)-\left(2+\frac{2}{\gamma^{2}}\right) \beta\right] .
\end{aligned}
$$

The procedure for Breit-Wheeler muon generation is first to transform from the lab frame to the CM frame of the photons and hence also of the muons, then evaluate Eq. (17). Inversion of the differential cross section leads to determination of the pair production angles, and thus a complete determination of the kinetics of the final state. For the additional two processes, Landau-Lifshitz and BetheHeitler, the method of virtual quanta is employed. For muon production (as well as for incoherent pair production) the maximum virtuality of the photons is chosen as $E_{\mathrm{cm}} / 4$.

The muon production cross section should approach the well-tested incoherent pair production at high transverse momenta $p_{t}>m_{\mu} c$ since the mass contribution to the produced pair energies in the $\mathrm{CM}$ frame is negligible in this case. Thus, it was tested that the produced muon cross section coincides with the cross section for the incoherent pairs at high $p_{t}$ as seen in Fig. 18.

Although the muons have been allowed to emit synchrotron radiation in the simulation, they are not allowed to affect any beam particles or fields after they are produced.

The produced muon pairs were added to the tracking of beam pairs in GUINEA-PIG++. The tracking algorithm has not been fully verified for nonrelativistic particles and since muon pairs are produced with energies not much above their mass, one should use muon tracking in the current implementation of muon tracking with caution. Therefore the user can optionally store the produced pairs before beam-beam tracking, and the tracking of muons is optional. Since synchrotron radiation is highly unlikely 


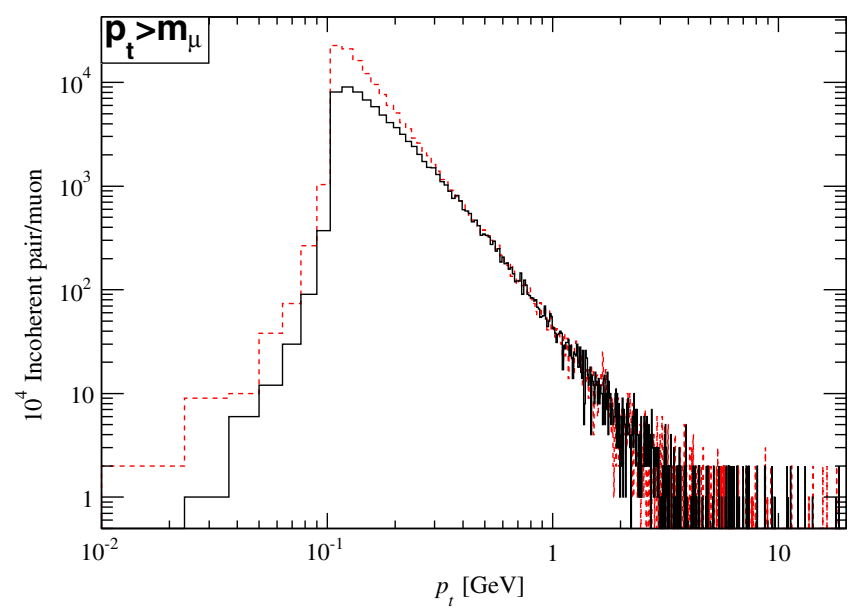

FIG. 18. Comparison of the transverse momentum of incoherent electrons and muons. Cross sections have been scaled up by $10^{4}$ to decrease simulation time. At production time, the cut $p_{t}>m_{\mu} c$ has been employed. Red dashed line indicates incoherent electrons. Black solid line indicates incoherent muons.

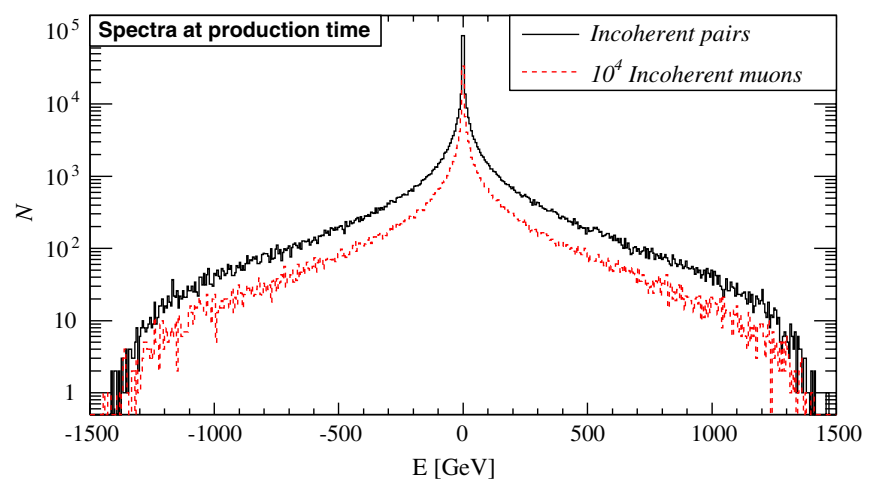

FIG. 19. Spectrum of incoherent muons at the time of production. The sign of the energy corresponds to the muon charge sign. The muon cross section is scaled up by a factor $10^{4}$, taking $\left(m_{\mu} / m_{e}\right)^{2}=4 \times 10^{4}$ into account, to decrease simulation time.

for muons, the beam-beam interaction hardly affects the muon spectrum.

For CLIC, the total number of produced muon pairs per bunch crossing is 12.5 . Of these particles 0.6 are produced through the Breit-Wheeler process, 8.0 through the Bethe-Heitler mechanism, and 3.9 from the LandauLifshitz process. Their spectrum is seen in Fig. 19, and their production angles can be seen in Fig. 1.

\section{B. $s$-channel muons}

To ensure that no other processes contribute significantly to the muon pair production cross section, it was checked that $s$-channel muons would not be of importance. The leading order processes are

$$
e^{+}+e^{-} \rightarrow \gamma \rightarrow \mu^{+}+\mu^{-}
$$

$$
e^{+}+e^{-} \rightarrow Z_{0} \rightarrow \mu^{+}+\mu^{-},
$$

where the intermediate particle is a virtual gauge boson. The first process gives rise to an $s^{-1}$ dependent cross section while the second process gives rise to the familiar Breit-Wigner resonance around the $Z_{0}$ mass of $91 \mathrm{GeV}$.

The total $s$-channel cross section can be found in $[46,47]$. A very rough integration of the $1 / s$ shape in the range $30-150 \mathrm{GeV}$ reveals that the photon diagram contribution to the cross section in this range is $\approx 20 \mathrm{pb}$ while the $Z_{0}$ diagram contribution in this range is of the order of $1 \mathrm{nb}$.

Looking at the luminosity spectrum in Fig. 13, one clearly sees that there is not enough luminosity at CLIC in the vicinity of the $Z_{0}$ resonance to make it worthwhile to include $s$-channel diagrams in the simulation. An upper bound of the luminosity in the $Z_{0}$ range is $10^{-6} \mathrm{nb}^{-1}$ per bunch crossing, meaning that approximately $10^{-6}$ muons per bunch crossing would be produced via these mechanisms. Therefore, these events can safely be excluded from simulations.

\section{TERTIARY PHOTONS}

At present, several produced particles could provide excellent fast luminosity signals. These particles include beamstrahlung photons and large $p_{t}$ hadronic events [48]. Here, we propose another fast luminosity signal arising from optical photons.

Synchrotron radiation emitted from incoherent pairs share the large angles of these particles, and could potentially supply information about luminosity and/or reach unintended parts of the detector. Therefore, it was decided to simulate the creation and the properties of these "tertiary photons." Their kinematic information was saved to a separate output file of GUINEA-PIG++.

The angles of the tertiary photons can be found in Fig. 1, where the large production angles are apparent. In Fig. 20 is seen the azimuthal information for these particles, where their trajectories have been projected onto a transverse

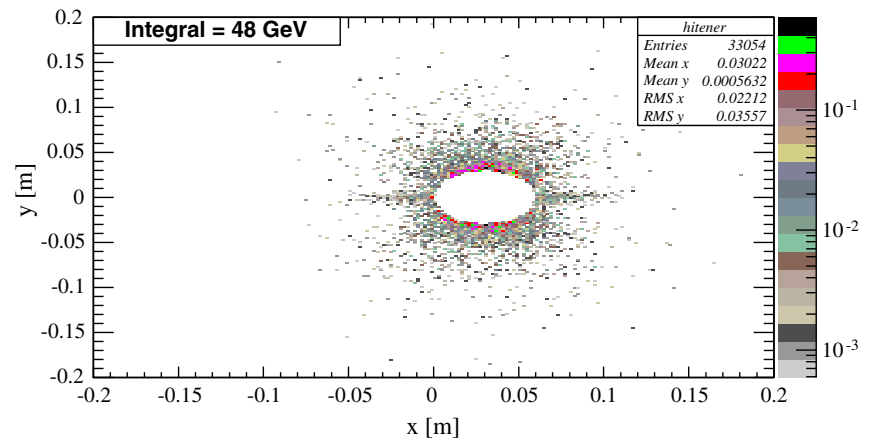

FIG. 20. Spatial distribution of energy deposited by the tertiary photons at a transverse plane $3 \mathrm{~m}$ from the IP. Only photons with production angles above $20 \mathrm{mrad}$ are displayed here. 
plane $3 \mathrm{~m}$ from the IP. The $10 \mathrm{mrad}$ width of the beam pipe has been cut away revealing that the energy deposition in the detector is very small and in the vicinity of $48 \mathrm{GeV}$ per bunch crossing. This number should be compared to $27 \mathrm{TeV}$ for the incoherent pairs, $2200 \mathrm{GeV}$ for the coherent pairs and $880 \mathrm{GeV}$ for direct trident pairs, respectively.

The energy of the tertiary photons can be very low as seen in Fig. 21. In fact, the spectrum extends to energies below those of optical photons. These photons are readily detected, and could provide information about the instantaneous luminosity. In Fig. 22, the rather strong correlation between the tertiary photon yield and luminosity is seen. Since the process is partly incoherent and partly a result of the interaction with the field, the dependence of tertiary photons with some imperfection could potentially be quite complex. But there seems to be a direct proportionality between luminosity and tertiary photon counts. Furthermore they are sufficiently abundant for detection and hence also for fast luminosity monitoring.

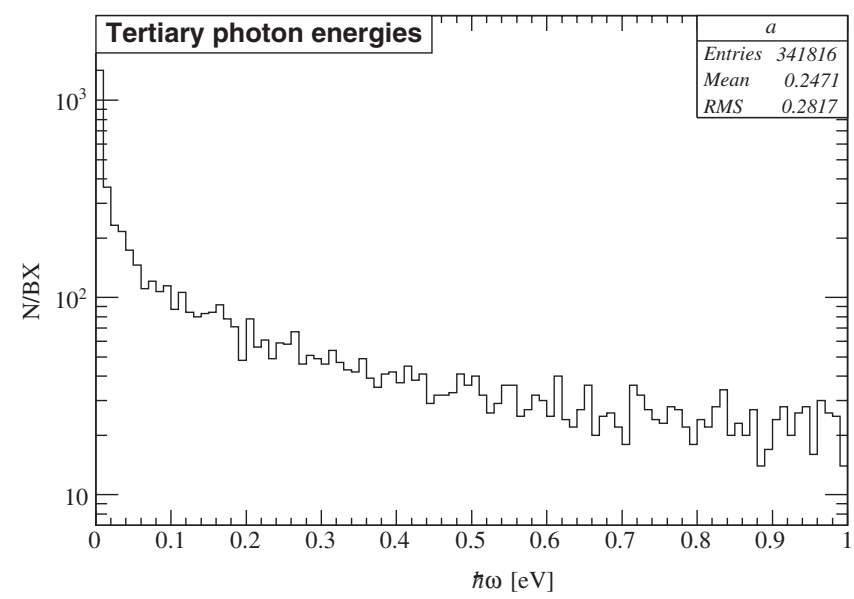

FIG. 21. Low-energy part of the tertiary photon spectrum.

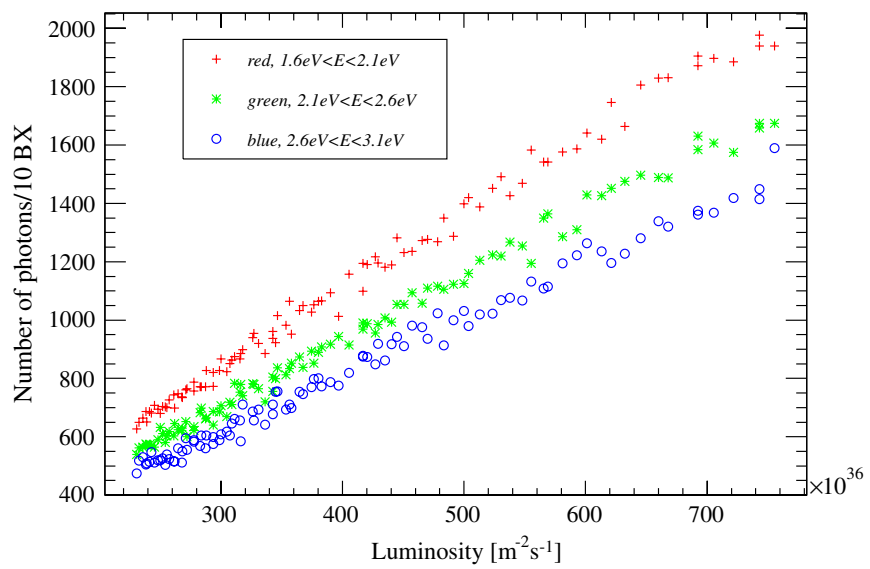

FIG. 22. Correlation between luminosity and number of tertiary photons (per 10 bunch crossings) when the size of one beam is varied. Each color in the optical region corresponds to an energy bin of $0.5 \mathrm{eV}$ width.
Since the tertiary photons are not affected by the solenoid field, the azimuthal information about their distribution could prove very useful. And there might be some useful spectral information to be found in the tertiary photons. This would be due to the fact that a larger average beam-beam field, induced by some imperfection and experienced by the incoherent pairs, would increase the critical frequency of synchrotron radiation.

The low-energy photon background from other pairs at the interaction point would have to be determined in order to assess the feasibility of using the tertiary photons as a luminosity signal. Especially the direct trident pairs would be able to emit light at rather large angles. Since direct trident pairs in CLIC are more abundant than incoherent pairs, there is a chance that photons from these particles would create a large background. However, synchrotron photons from these particles will not extend to very large angles, so in all likeliness there is an angular cut above which only tertiary photons would be seen. Searches for background radiation would also include an estimate of the synchrotron yield from the interaction with the detector solenoid field. If these particles turn out to be of interest, the inclusion of an emission angle, as opposed to the current implementation, where the photons have the direction of the emitting electron, would be essential.

Finally, the vertex detectors are in all likeliness not sensitive to these photons. However, the spectrum of tertiary photons is very wide and some frequencies of the light could potentially trigger signals in them.

\section{CONCLUSIONS}

New processes have been implemented and simulated in GUINEA-PIG. These new additions have been tested along with previously implemented ones. In particular, a Weizsäcker-Williams calculation of the direct trident process that is valid in the quantum mechanical as well as in the intermediate $\Upsilon \approx 1$ regime has been implemented. This simulation has been applied to beam-beam simulations relevant for the next generation linear colliders. The impact on any proposed $3 \mathrm{TeV}$ CLIC design is small when compared to other processes of $e^{+} e^{-}$pair creation but may become significant in the limit of short bunches. The kinematic characteristics of the produced particles are not unlike those of sequential tridents (coherent pairs). Because the relative contribution of the direct tridents to the postcollisional bunch charge is small, this will mean that they are most likely difficult to distinguish from the coherent pairs in a detector without varying the bunch length. Depolarization due to beam-beam effects is below the percent level in the luminosity peak for CLIC at $3 \mathrm{TeV}$. The explanation is partly due to diminishment of the electron anomalous magnetic moment and partly due to lack of beamstrahlung from the most energetic particles. Approximately $12.5 \mu \mathrm{m}$ will be emitted per bunch crossing 
due to binary collisions which have been cross-checked with high $p_{t}$ results of incoherent pairs.

Incoherent pairs will emit synchrotron in the visible regime that extends to large polar angles. As optical photons are easily manipulated, they might be efficiently detected and used to monitor luminosity effectively and fast.

[1] ATLAS Collaboration, Phys. Lett. B 716, 1 (2012).

[2] CMS Collaboration, Phys. Lett. B 716, 30 (2012).

[3] D. Schulte, Doctorate, Universität Hamburg, 1996.

[4] C. Rimbault et al., Report No. EuroTeV-Report-2007-056, 2007.

[5] J. Esberg, Ph.D. thesis, Aarhus University, 2012.

[6] CLIC Collaboration, CERN Report No. CERN-12-007, 2012.

[7] R. Noble, Nucl. Instrum. Methods Phys. Res., Sect. A 256, 427 (1987).

[8] P. Chen, Report No. SLAC-PUB-5914, 1992.

[9] P. Chen and K. Yokoya, Phys. Rev. D 38, 987 (1988).

[10] K. Yokoya and P. Chen, Lect. Notes Phys. 400, 415 (1991).

[11] K. Yokoya, Nucl. Instrum. Methods Phys. Res., Sect. A 251, 1 (1986).

[12] K. K. Andersen, J. Esberg, H. Knudsen, H. D. Thomsen, U. I. Uggerhøj, P. Sona, A. Mangiarotti, T. J. Ketel, A. Dizdar, and S. Ballestrero (CERN NA63 Collaboration), Phys. Rev. D 86, 072001 (2012).

[13] K. Yokoya and P. Chen, SLAC-PUB-4692, 1988; K. Yokoya, User's Manual of CAIN, Version 2.35 (2003).

[14] T. Erber, Rev. Mod. Phys. 38, 626 (1966).

[15] P. Chen and V. I. Telnov, Phys. Rev. Lett. 63, 1796 (1989).

[16] K. A. Thompson and P. Chen, AIP Conf. Proc. 472, 260 (1999).

[17] J. Esberg and U. I. Uggerhøj, J. Phys. Conf. Ser. 198, 012007 (2009).

[18] J. Esberg, K. Kirsebom, H. Knudsen, H. D. Thomsen, E. Uggerhøj, U. I. Uggerhøj, P. Sona, A. Mangiarotti, T. J. Ketel, A. Dizdar, M. M. Dalton, S. Ballestrero, and S. Connell (CERN NA63 Collaboration), Phys. Rev. D 82, 072002 (2010).

[19] R. Blankenbecler and S. D. Drell, Phys. Rev. D 36, 277 (1987).

[20] U. I. Uggerhøj, Rev. Mod. Phys. 77, 1131 (2005).

[21] V. N. Baier, V. M. Katkov, and V. M. Strakhovenko, Sov. J. Nucl. Phys. 53, 632 (1991).

[22] J. C. Kimball and N. Cue, Phys. Rep. 125, 69 (1985).

[23] K. A. Thompson and P. Chen, Report No. SLAC-PUB7776, 1998.
[24] V. N Baier, V. M. Katkov, and V. M Strakhovenko, Electromagnetic Processes at High Energies in Oriented Single Crystals (World Scientific, Singapore, 1998).

[25] D. White, Phys. Rev. D 24, 526 (1981).

[26] D. White, Phys. Rev. D 26, 10 (1982).

[27] V. I. Ritus, Nucl. Phys. B44, 236 (1972).

[28] V. B. Berestetskii, E. M. Lifschitz, and L. P. Pitaevskii, Relativistic Quantum Theory, Course of Theoretical Physics Vol. 4 (AIP, New York, 1971), part 1.

[29] V. M. Budnev, I. F. Ginzburg, G. V. Meledin, and V. G. Serbo, Phys. Rep. 15, 181 (1975).

[30] K. Kirsebom et al., Nucl. Instrum. Methods Phys. Res., Sect. B 135, 143 (1998).

[31] V. N. Baier and V. M. Katkov, Phys. Lett. A 346, 359 (2005).

[32] V. N Baier and V. M. Katkov, Sov. Phys. Dokl. 17, 1068 (1973).

[33] A. Miyamoto, M. Stanitzki, H. Weerts, and L. Linssen Report No. CERN-12-008, 2012.

[34] B. Dalena, J. Esberg, and D. Schulte, 2011 Proceedings of the International Workshop on Future Linear Colliders, arXiv:1202.0563.

[35] M. Woods, Report No. SLAC-PUB-8397, 2000.

[36] T. Nishitani et al., J. Appl. Phys. 97, 094907 (2005).

[37] X. Artru, R. Chehab, M. Chevallier, and V. Strakhovenko, Phys. Rev. ST Accel. Beams 6, 091003 (2003).

[38] L. H. Thomas, Nature (London) 117, 514 (1926).

[39] V. Bargmann, L. Michel, and V. L. Telegdi, Phys. Rev. Lett. 2, 435 (1959).

[40] K. Yokoya and P. Chen, Report No. SLAC-PUB-4692, 1988.

[41] A. Ferrari, V. Ziemann, R. B. Appleby, and M. Salt, Phys. Rev. ST Accel. Beams 12, 021001 (2009).

[42] V. N. Baier, V. M. Katkov, and V. M. Strakhovenko, Lett. Nuovo Cimento 15, 5 (1976).

[43] C. Rimbault et al., Report No. EUROTeV-report-2008-066, 2008.

[44] K. Kirsebom, U. Mikkelsen, E. Uggerhøj, K. Elsener, S. Ballestrero, P. Sona, and Z. Z. Vilakazi, Phys. Rev. Lett. 87, 054801 (2001).

[45] J. Esberg, D. Schulte, U. I. Uggerhøj, and B. Dalena, CLIC-Note-2011-934, 2011.

[46] F. A. Berends, R. Kleiss, and S. Jadach, Nucl. Phys. B202, 63 (1982).

[47] U. Becker, Nucl. Phys. B, Proc. Suppl. 19, 333 (1991).

[48] B. Dalena, J. Barranco, A. Latina, E. Marin, J. Pfingstner, D. Schulte, J. Snuverink, R. Tomás, and G. Zamudio, Phys. Rev. ST Accel. Beams 15, 051006 (2012). 